\title{
Measurements of Integration Gain for the Cospas-Sarsat system from Geosynchronous Satellites
}

Elizabeth Klein-Lebbink, Sr Member

James Christo

Dr. Robert Peters, Member

Xuan Nguyen

ICSSC 2015 


\section{Overview of Search and Rescue, (SAR)}

- 43 Countries participate, NOAA-SARSAT is a part of that for the USA

- Detects and locates aviators, mariners, hikers and mountain climbers in distress

- Each carry distress beacons which get activated when they run into trouble

- Various satellites relay the signals from the distress beacons to ground stations around the world

- Existing system is multilayered, relies on LEO (low earth orbit) and GEO (geosynchronous earth orbit) satellites, and in the near future MEO (medium earth orbit) satellites including GPS

- Discussed here are measurements made with the GEO ground user terminal (GEOLUT) in the SARLab at the NASA Goddard campus

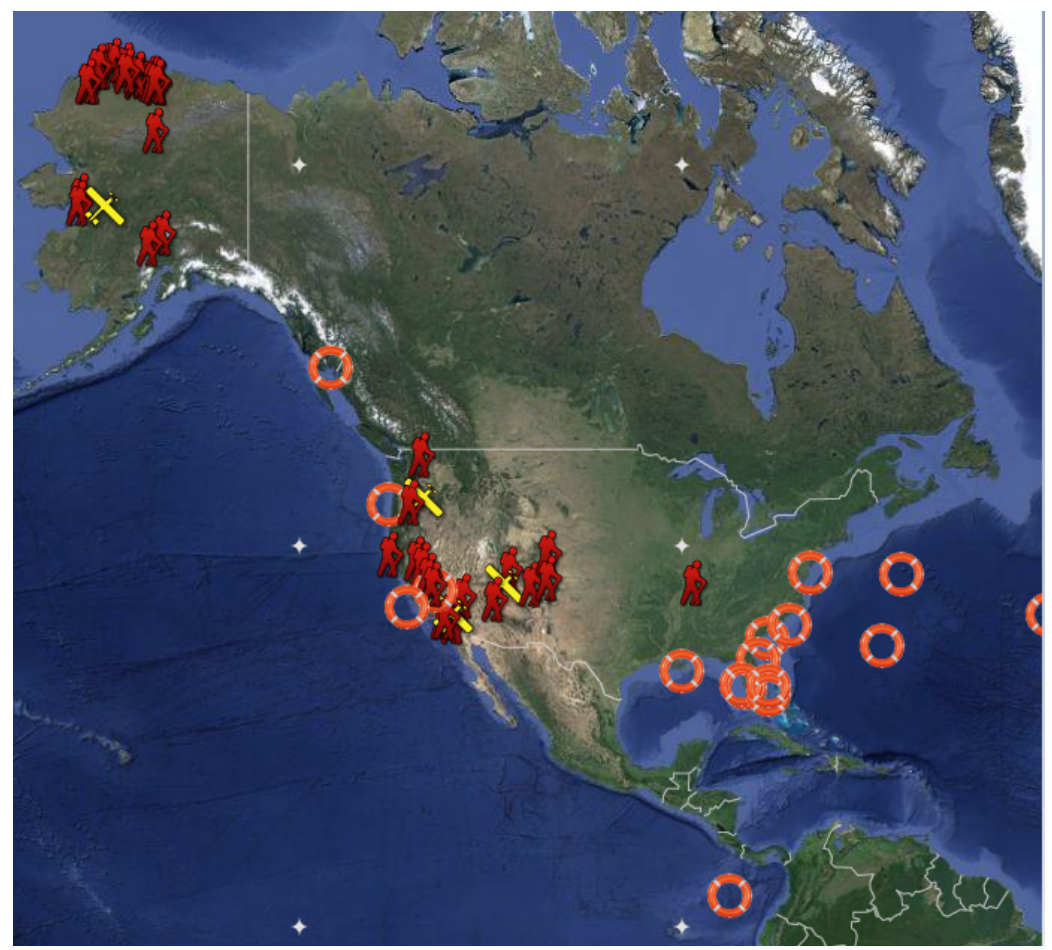

Western Hemisphere SARSAT rescues in 2014 


\section{Overview}

\section{COSPAS-SARSAT System Overview}

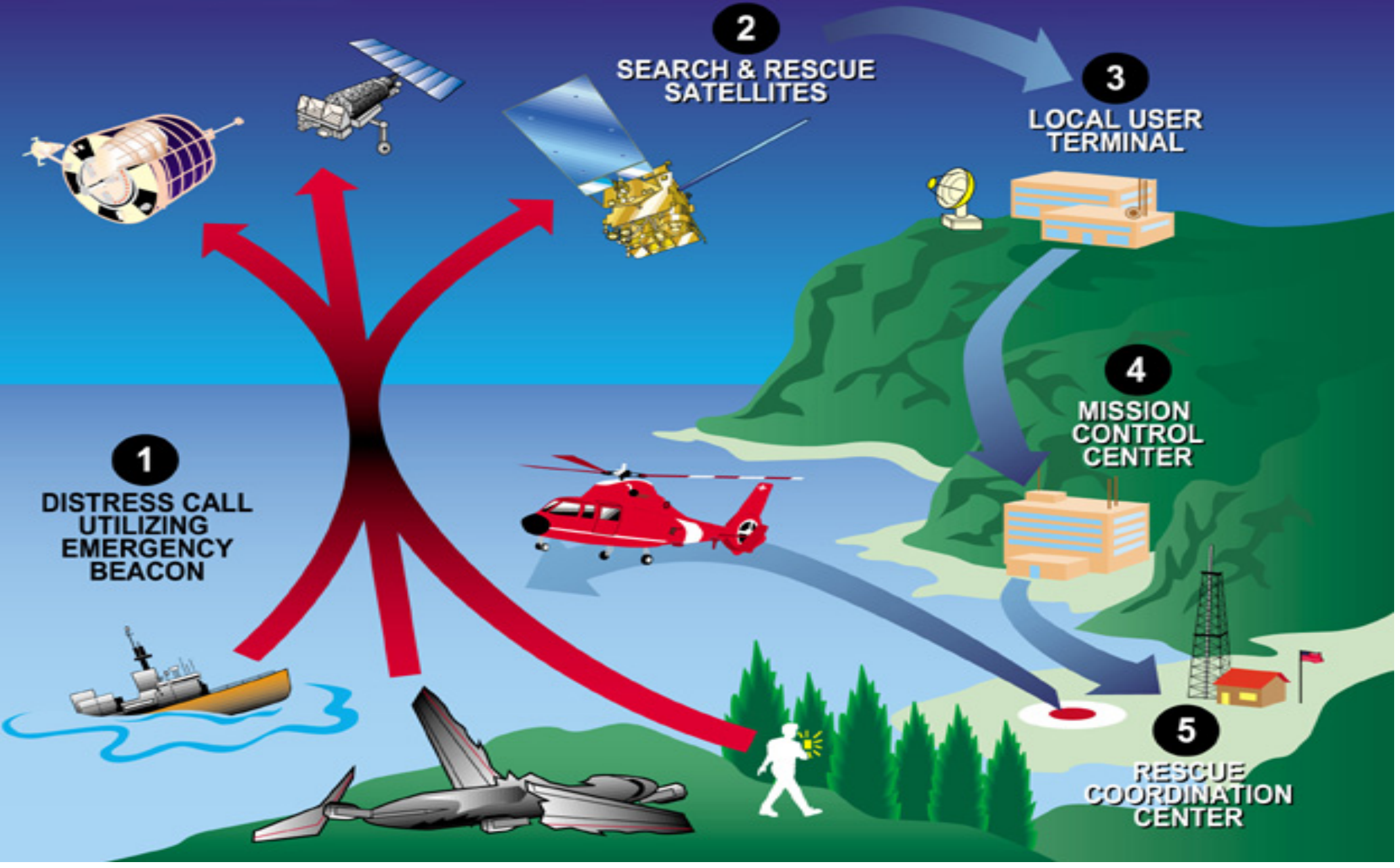




\section{Test Description}

- Tests were conducted at the Search and Rescue Laboratory (SARLab) at NASA Goddard

- Used a beacon simulator, spacecraft engineering design units (EDUs), and a Geo Local User Terminal (GEOLUT) to make a high fidelity test of integration gain and overall compatibility with the new spacecraft configuration

- The data desired was the percentage of successful message decodings vs. the GEOLUT received $\mathrm{C} / \mathrm{No}$.

- The test was set up to first calibrate the $\mathrm{C} / \mathrm{No}$ of the radio frequency link which was critical; although the GEOLUT reports a $\mathrm{C} / \mathrm{No}$, its accuracy was unknown 


\section{Test Set up}

- Test procedure run first using the SARLab's satellite simulator and then through the engineering satellite transponder equipment (EDU) with and without message integrations to determine the overall integration gain

- GOES-R series spacecraft is the first GEO s/c that does not have on board re-modulation

- Comparison were also performed with the GOES-13 operational spacecraft which does have an on board re-modulator 


\section{Test set up}

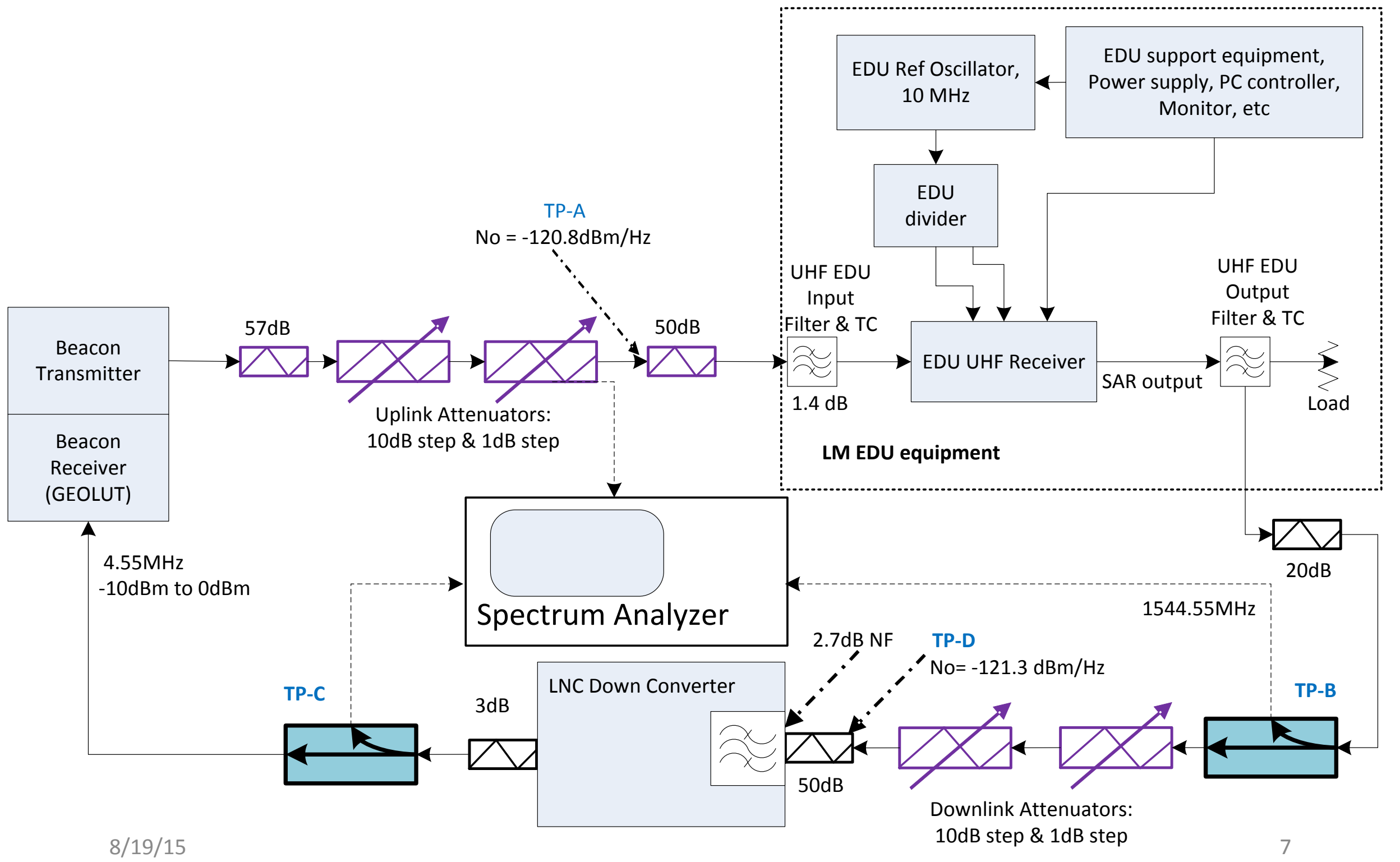




\section{Message Counting for Integration}

- There are two options on how the integrated messages are counted, as there are no Cospas-Sarsat standards or requirements in this area

- The first method is to count all resulting messages from the integration process. For example, if 8 received bursts occur prior to a successful message decoding, then one counts the 8 resulting digital messages as 8 message acquisitions. Note the GEOLUT fills in each of the 8 bursts with the identical successfully decoded message

- The second method is to count only one resulting message. Using the above example, there is only one successful decoding of the 8 integrated bursts one counts only the one successful decoding as the one message acquisition. While the other 7 bursts will have the same decoded digital message in this GEOLUT, they are not independent of the one countable message burst. 


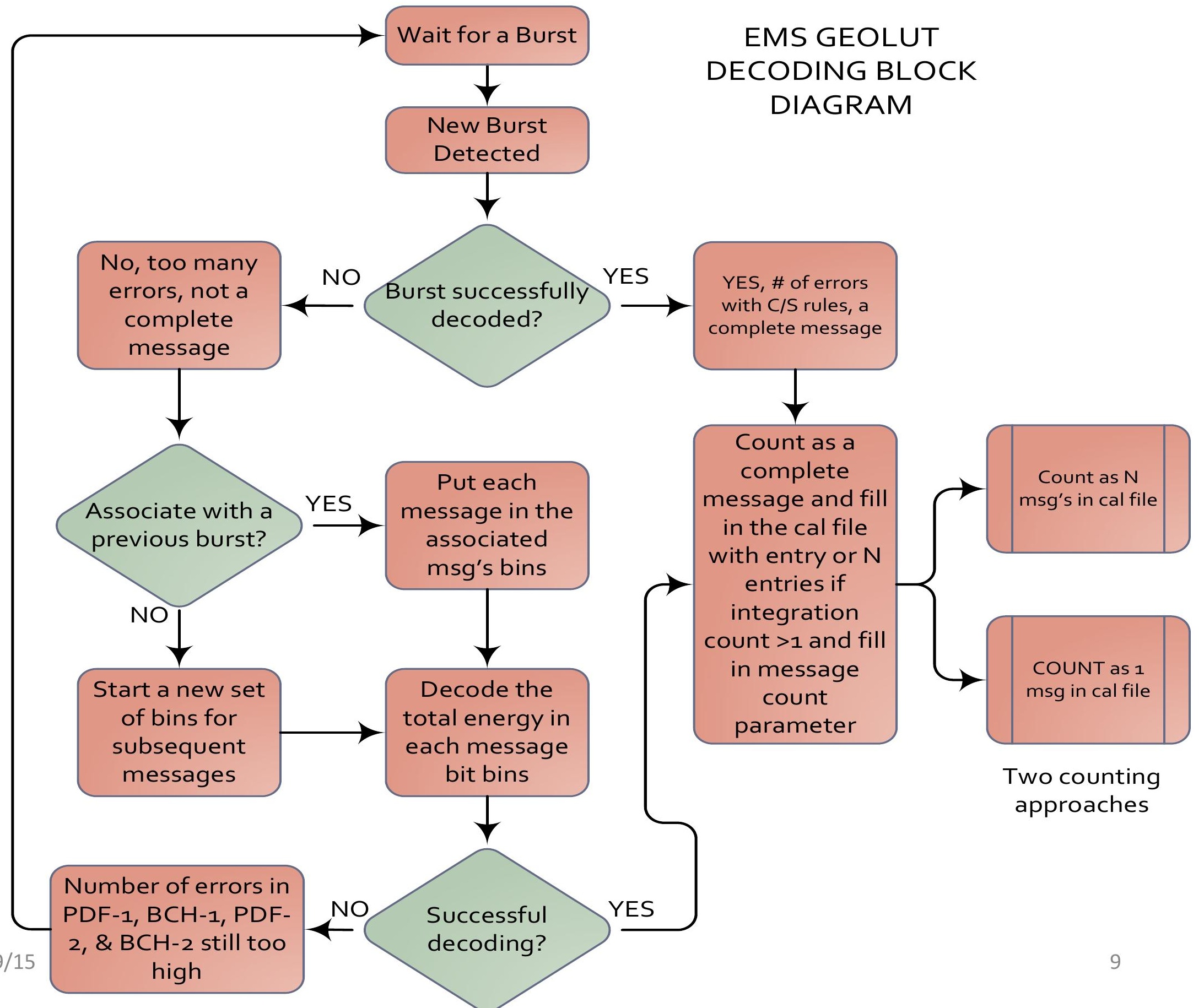




\section{Results}

Total message count comparison using Method 1

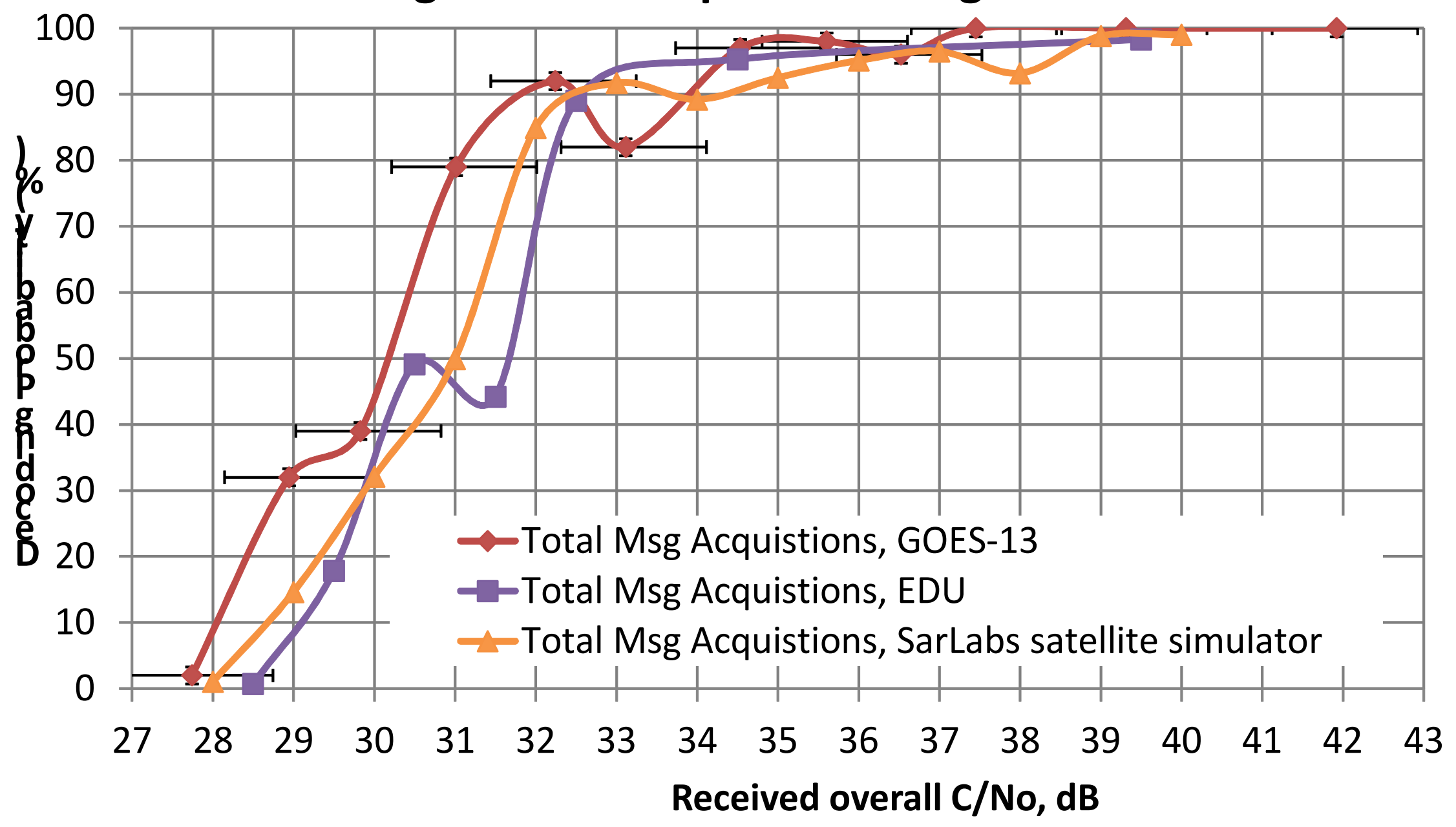


Integration results from the EDU measurements using method one - counting all integrated messages as separate messages

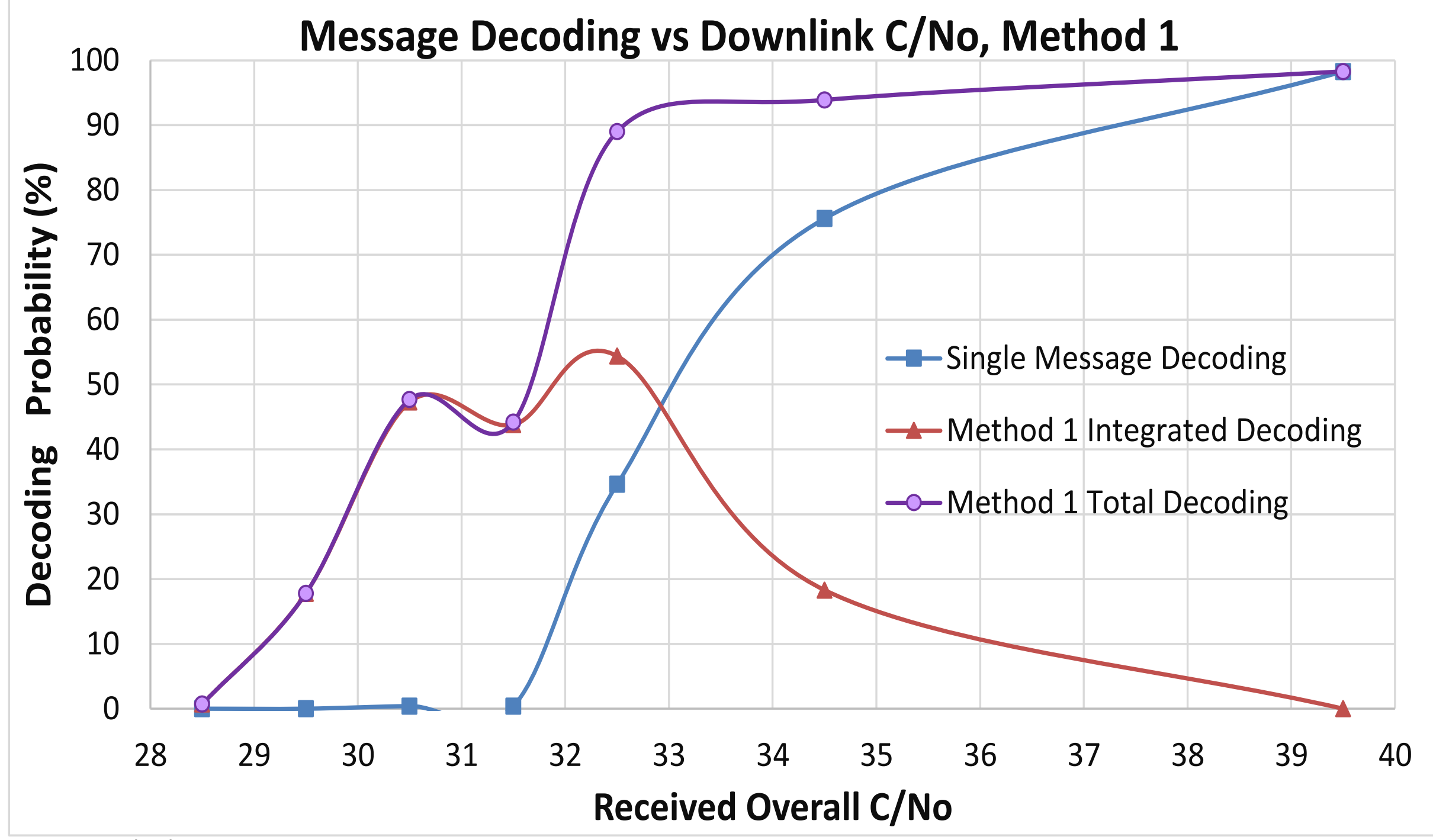




\section{Integration results from the EDU measurements using method two - counting all integrated messages as one message acquisition}

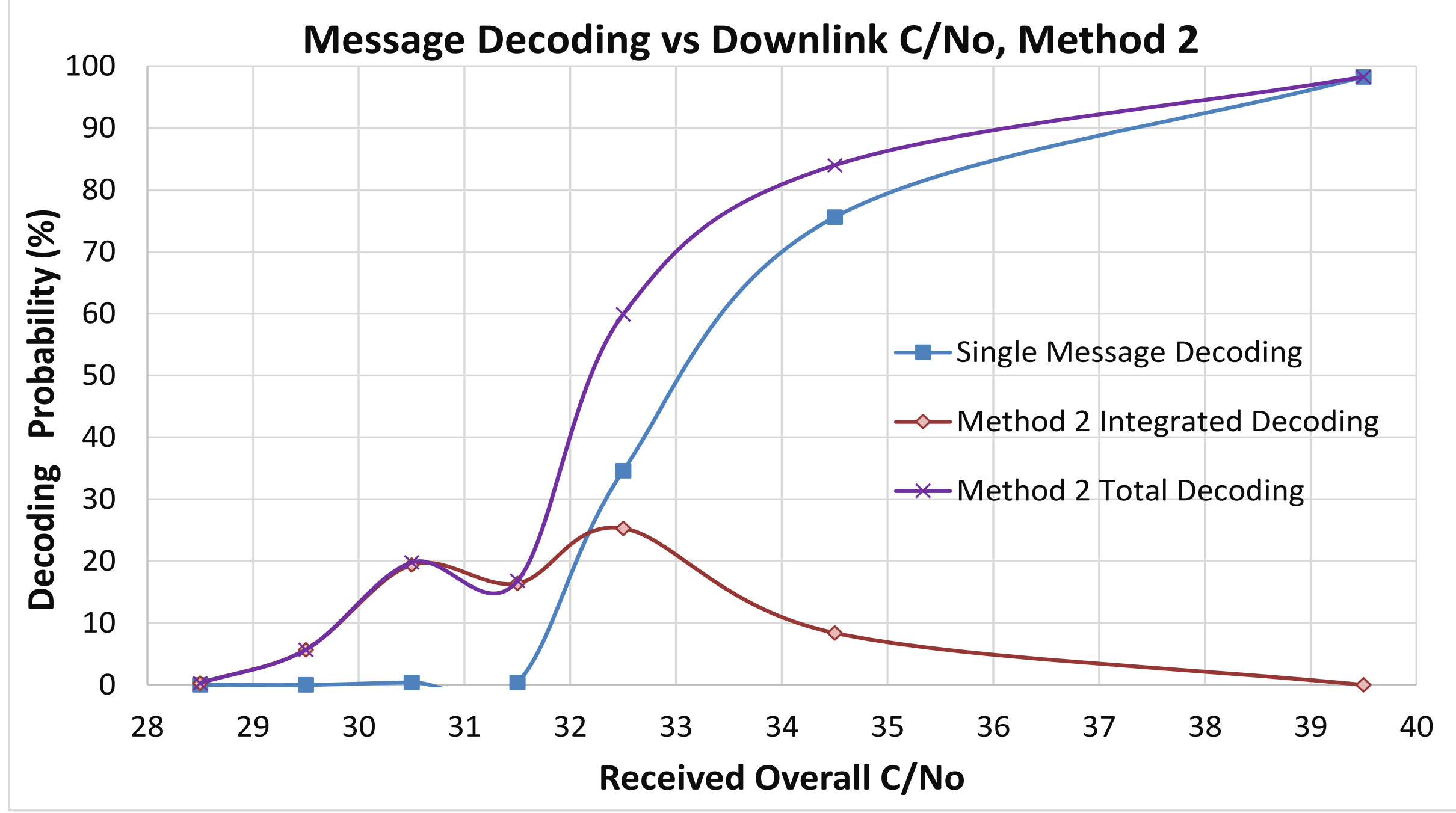




\section{Conclusions}

- A Series of firsts!

- First time the SAR transponder has ever tested prior to launch, first test through a bent pipe satellite transponder (no on-board re-modulation), and the first measurement of integration gain.

- Tests confirmed that the required performance will be met

- SARLab developed new tool - a satellite simulator which proved its value in the procedure development process 


\section{Further study}

- Since the message is made of coded and uncoded components, calculating the theoretical BER for a given C/No is not simple

- The major LUT vendors claim not to have run performance tests. Cospas-Sarsat has minimal specifications on how it should be accomplished and reported. In the development leading up to the EDU test, the SARLab developed two possible approaches counting the integrated bursts.

- A final note to reiterate that this conclusion only applies to the GEOLUTs in use by the SARLab. Other vendors GEOLUTs may present the data differently and may or may not show all the bursts that went into an integrated detection. This issue is external to the GOES-R project and deserves further study by Cospas-Sarsat to ascertain which approach will yield more realistic result when the satellite is launched. 